\title{
Causal Attributions of Ghanaian Industrial Workers for Accident Occurrence
}

\author{
SETH AYIM GYEKYE \\ Department of Social Psychology \\ University of Helsinki \\ Helsinki, Finland
}

\author{
SIMO SALMINEN ${ }^{1}$ \\ Department of Occupational Safety \\ Finnish Institute of Occupational Health \\ Helsinki, Finland
}

\begin{abstract}
This study confirms the self-defensive attribution hypothesis on causal attributions of accidents in Ghana's work environment. In this investigation, Ghanaian industrial workers and their supervisors assigned causality to industrial accidents, and their responses were compared. The results showed that the victims attributed their accidents to external causes to a greater extent than did the supervisors, and to internal causes to a lesser extent than did the supervisors. This finding reflects the tendency toward self-protective bias, whereby people tend to project blame for their failures onto external circumstances.
\end{abstract}

In the execution of job assignments, employees often encounter negative performance outcomes that lead to information search and attribution formulation. These causal explanations help people to find remedies that are used to prevent future accidents from happening. The importance of being able to explain and predict such accidents has led to a number of studies on attribution explorations. However, the research results on causal attributions have often provided evidence of self-serving and ego-protecting biases.

These attributional distortions are rather common in novel and ambiguous situations (Wong \& Weiner, 1981), where one is faced with multiple causal agents, as in the case of industrial accidents (DeJoy, 1985, 1990; Turner \& Pidgeon, 1997). As industrial accidents tend to afford fertile ground for causal and responsibility attributional distortions and biases, the work environment therefore seems to be the appropriate domain to examine evidence of causal attribution biases and distortions. An example of such attributional distortion occurs when people make use of self-protective mechanisms to project blame for their personal failures onto external circumstances. This has been labeled the selfdefensive attribution hypothesis (Shaver, 1970a; Walster, 1966).

${ }^{1}$ Correspondence concerning this article should be addressed to Simo Salminen, Finnish Institute of Occupational Health, Department of Occupational Safety, Topeliuksenkatu 41b, FIN-00250 Helsinki, Finland. E-mail: simo.salminen@ttl.fi

\section{4}

Journal of Applied Social Psychology, 2004, 34, 11, pp. 2324-2342.

Copyright $(\mathcal{C} 2004$ by V. H. Winston \& Son, Inc. All rights reserved. 


\section{The Self-Defensive Attribution Hypothesis}

The defensive attribution hypothesis (Shaver, 1970a) originated from a study by Walster (1966) and assumes that participants in an accident process tend to explain the accident occurrence in a way that minimizes their personal responsibility. They do this by externalizing causality and invoking alternative explanations to protect their self-esteem (Zuckerman, 1979). Thus, self-defensive attribution is often described in the literature as a notion of self-protective attributional distortion through which people deny or minimize the implication of their responsibility in failure events (Blass, 1996; Burger, 1981).

The defensive attribution hypothesis has been confirmed in laboratory studies (Chaikin \& Darley, 1973; Shaw \& Skolnick, 1971) and has received support from empirical research in the work environment (Kouabenan, 1985; Kouabenan, Gilibert, Medina, \& Bouzon, 2001; Salminen, 1992). These and other studies have indicated that both accident victims and supervisors tend to attribute the accident occurrence to causal factors in a way that diminishes their own responsibility. Supervisors, more than their subordinates, tend to attribute workers' errors (Mitchell \& Kalb, 1981; Mitchell \& Wood, 1980) and serious accidents (DeJoy, 1985, 1987) to factors internal to the workers. Reviews by Vidmar and Crinklaw (1974) and by Burger (1981) have supported this stance.

Although promising, the research on defensive attribution has been developed and tested primarily with Western samples only, which represent roughly $27 \%$ of humankind. To our knowledge, no study has examined this phenomenon in a non-Western culture. Consequently, there is a paucity of cross-cultural replicative studies. Given the important differences between Western and Eastern cultures (Hofstede, 1980, 1991; Triandis, 1995), we consider it essential to test the self-defensive attribution hypothesis in an African culture; specifically, in Ghana's work environment.

\section{Method}

\section{Participants and Material}

The current study is part of a larger comparative analysis that examined the causal and responsibility attributions for accident occurrence between Ghanaian and Finnish industrial workers (Gyekye, 2001). The participants were actual victims, coworkers and supervisors involved in workplace accidents. They were to attribute causality to specified accidents in which they had been involved. They comprised 320 Ghanaian industrial workers from mines and factories: 121 were accident victims, 117 were coworkers, and 82 were supervisors. The average ages were as follows: accident victims, 37 years $(S D=9.71)$; 
coworkers, 35 years $(S D=8.22)$; and supervisors, 44 years $(S D=6.80)$. All of the accident victims and supervisors were men, whereas $13.7 \%$ of the coworkers were women.

The following steps were taken to contact respondents and to arrange for interviews. Letters requesting permission to undertake accident investigation as part of an academic program were sent to the Chief Inspectorates of Factories and Mines in Ghana. The response was prompt and encouraging. A list of industrial accidents that had been reported was provided. To elicit a fair recall of the accident causality and occurrence, industrial workers who had been involved in or witnessed accident occurrences within the year or the previous year were selected as respondents. To ensure the accident severity dimension that is crucially needed in self-defense attributions (Kouabenan et al., 2001; Shaver, 1970b), all reported cases in this study were of those classified as serious by the Factories and Mines Inspectorates. Temporary injuries in which victims were absent for less than 3 days of work activity were thus excluded from the data.

The questionnaire (Appendix) was part of an extended version of an earlier study on the Finnish work environment (Salminen, 1997). Participants responded to 30 questions that employed a 5-point response format ranging from 1 (very little) to 5 (very much). These were causal explanations generated for the accident occurrence and classified as factors reflecting the dispositional qualities of the accident victims (internal factors), or those of the situational and environmental factors (external factors). This allowed respondents to rate their own attributions on dimensions of external and internal causality factors. In effect, all attributions for the accident causality were coded as being either internal or external. The higher a participant scored, the greater the attribution that he or she made for that causal factor.

The presentation of the interview was such that respondents felt reasonably comfortable about expressing personal beliefs (religious and otherwise), values, and work experiences. The duration varied from 15 to $20 \mathrm{~min}$, depending on the context in which they were conducted and on respondents' level of education. The questionnaire interview was presented in English. Where respondents were illiterate or semiliterate and had problems understanding English, the services of an interpreter were sought and the local dialect was used. Supervisors were educationally sound and filled in the questionnaire on their own.

The interview was administered individually. This ensured anonymity and the promise that all responses would be treated with the strictest confidentiality. To ensure accuracy of responses, it was also emphasized that the study was part of an academic work and that no person affiliated with their organization was involved in any way. Because of the nature of the study, special interest was paid to victims' and supervisors' causality attributions. The role of coworkers in selfdefense attributions has been undertaken in a separate study. 
Table 1

Means for Ghanaian Victims, Coworkers, and Supervisors on External and Internal Measures

\begin{tabular}{|c|c|c|c|c|c|c|}
\hline \multirow[b]{3}{*}{ Measure } & \multicolumn{6}{|c|}{ Group } \\
\hline & \multicolumn{2}{|c|}{$\begin{array}{l}\text { Victims } \\
(n=121)\end{array}$} & \multicolumn{2}{|c|}{$\begin{array}{c}\text { Coworkers } \\
(n=117)\end{array}$} & \multicolumn{2}{|c|}{$\begin{array}{l}\text { Supervisors } \\
\quad(n=82)\end{array}$} \\
\hline & $M$ & $S D$ & $M$ & $S D$ & $M$ & $S D$ \\
\hline External scale & 49.88 & 7.62 & 47.03 & 6.57 & 38.35 & 6.39 \\
\hline Internal scale & 34.01 & 5.99 & 36.87 & 7.45 & 47.81 & 9.52 \\
\hline
\end{tabular}

\section{Questionnaire Scoring and Reliability}

The 30 questions on the questionnaire were computed to give total scores for external and internal causes for each participant. This resulted in responses to each item being placed in one of five categories. As a result, each individual had item-by-item scores, as well as two total scores. This allowed comparison of participants on both the external and internal causal factors scales.

Internal coherence and reliability for the external and internal causal scales were tested with Cronbach's alpha coefficient. Acceptable coefficients of .89 and .79 were obtained for the external and internal causal factors, respectively, indicating high inter-item consistency. To improve the validity and reliability of the research instrument, the questionnaire was pilot-tested in the African community in Helsinki, Finland (among Ghanaian and Nigerian students who also work part-time and are thus familiar with accidents in the work environment). This provided feedback on the clarity of the questions and the overall presentation of the questionnaire.

\section{Procedure}

To arrive at the intended analyses, three sets of statistical analyses were conducted. First, the mean scores were computed (Table 1) and subjected to a oneway ANOVA to determine differences of statistical significance on both the external and internal causal factors scale. Second, $t$-test values were employed to further test for statistically significant differences between victims' and supervisors' causality attributions on the internal and external causal factors scale. The third part of the analyses involved an item-by-item comparison between victims' and supervisors' causal attributions on the external (Table 2) and internal causal scales (Table 3 ) via $t$-test values. 
Table 2

Means for Victims and Supervisors on External Causal Factors Scale

\begin{tabular}{|c|c|c|c|c|c|c|c|}
\hline \multirow[b]{2}{*}{ Measure } & \multicolumn{3}{|c|}{ Victims } & \multicolumn{3}{|c|}{ Supervisors } & \multirow[b]{2}{*}{$p<$} \\
\hline & $M$ & $S D$ & $N$ & $M$ & $S D$ & $N$ & \\
\hline Low wages & 3.46 & 1.48 & 121 & 3.89 & 1.00 & 82 & .05 \\
\hline Saving of time and trouble & 4.15 & 0.80 & 120 & 1.78 & 0.91 & 82 & .001 \\
\hline Work overload & 4.35 & 0.64 & 120 & 2.04 & 1.01 & 82 & .001 \\
\hline Defective equipment & 2.86 & 1.39 & 115 & 2.64 & 1.29 & 82 & $n s$ \\
\hline Inadequate training & 2.88 & 1.27 & 120 & 1.90 & 0.91 & 82 & .001 \\
\hline Pressure from management & 4.23 & 0.85 & 120 & 2.78 & 1.04 & 82 & .001 \\
\hline Coworker's fault & 2.56 & 1.28 & 116 & 2.35 & 1.09 & 82 & $n s$ \\
\hline Effect of medication & 2.38 & 1.11 & 105 & 2.84 & 0.52 & 70 & .001 \\
\hline Operational procedures & 4.21 & 0.74 & 121 & 2.67 & 0.79 & 80 & .001 \\
\hline Misassignment & 2.89 & 1.35 & 121 & 2.23 & 1.10 & 82 & .001 \\
\hline Curse/spell/witchcraft & 3.01 & 1.26 & 96 & 2.73 & 0.62 & 75 & $n s$ \\
\hline Religious faith & 3.05 & 1.24 & 95 & 2.80 & 0.51 & 75 & $n s$ \\
\hline Poor housekeeping & 3.44 & 1.25 & 121 & 2.25 & 1.07 & 82 & .001 \\
\hline Lack of appropriate gear & 3.55 & 1.39 & 121 & 2.51 & 1.25 & 82 & .001 \\
\hline $\begin{array}{l}\text { Ambiguity and task } \\
\text { difficulty }\end{array}$ & 3.32 & 1.14 & 121 & 2.58 & 0.81 & 82 & .001 \\
\hline
\end{tabular}

Note. Response options: $1=$ very little $; 2=$ quite little $3=$ neutral $; 4=$ quite much $; 5=$ very much.

\section{Results}

The one-way ANOVA indicates that all three subgroups perceived significant differences on the external causal factors scale, $F(2,208)=51.53, p<.001$; as well as on the internal causal factors scale, $F(2,307)=83.87, p<.001$. The means for the three subgroups indicate that accident victims, contrary to their supervisors, attributed accident causality more to factors that were external to the victims and less to internal factors.

\section{Comparative Analyses}

Accident victims and supervisors. The results of the $t$-test evaluations on the external and internal causal factors scale confirm the existence of statistically 
Table 3

Means for Victims and Supervisors on Internal Causal Factors Scale

\begin{tabular}{|c|c|c|c|c|c|c|c|}
\hline \multirow[b]{2}{*}{ Measure } & \multicolumn{3}{|c|}{ Victims } & \multicolumn{3}{|c|}{ Supervisors } & \multirow[b]{2}{*}{$p<$} \\
\hline & $M$ & $S D$ & $N$ & $M$ & $S D$ & $N$ & \\
\hline Lack of skill & 1.67 & 0.85 & 121 & 2.78 & 1.35 & 82 & .001 \\
\hline Professional pride & 1.92 & 0.90 & 121 & 3.35 & 1.36 & 82 & .001 \\
\hline Lapse in attention & 1.99 & 1.26 & 121 & 3.59 & 1.29 & 82 & .001 \\
\hline Misperception & 2.15 & 1.04 & 121 & 3.43 & 1.26 & 82 & .001 \\
\hline Misconduct & 2.32 & 1.13 & 120 & 3.69 & 1.40 & 82 & .001 \\
\hline $\begin{array}{l}\text { Lack of adequate } \\
\text { comprehension }\end{array}$ & 2.19 & 1.00 & 121 & 3.32 & 1.30 & 82 & .001 \\
\hline Risky work habit & 2.19 & 0.93 & 120 & 2.91 & 1.38 & 82 & .001 \\
\hline Inexperience & 1.90 & 0.87 & 121 & 3.35 & 1.24 & 82 & .001 \\
\hline Carelessness & 1.96 & 0.93 & 121 & 3.92 & 1.18 & 82 & .001 \\
\hline Urge to show off & 1.97 & 0.96 & 120 & 2.84 & 1.26 & 82 & .001 \\
\hline Ignorance & 2.18 & 1.10 & 121 & 3.09 & 1.40 & 82 & .001 \\
\hline Sense of job security & 2.80 & 1.14 & 120 & 3.04 & 1.27 & 82 & $n s$ \\
\hline Mood, had a bad day & 3.02 & 1.24 & 121 & 2.08 & 1.07 & 82 & .001 \\
\hline Tiredness/exhaustion & 3.19 & 1.08 & 121 & 2.43 & 1.37 & 82 & .001 \\
\hline Deliberate, willful violation & 2.50 & 0.92 & 116 & 3.91 & 1.26 & 82 & .001 \\
\hline
\end{tabular}

Note. Response options: $1=$ very little $; 2=$ quite little $3=$ neutral $; 4=$ quite much $; 5=$ very much.

significant differences between the three subgroups. On the external scale, accident victims attributed significantly more causation to external and environmental factors than did their supervisors, $t(143)=9.89, p<.001$. On the internal scale, supervisors, as opposed to accident victims, attributed accident causality to factors that were internal to the accident victims to a statistically significant degree, $t(127)=-11.56, p<.001$.

Coworkers and supervisors. On the external scale, coworkers attributed the accidents significantly more to external factors than did their supervisors, $t(128)=7.62, p<.001$. On the internal scale, however, supervisors attributed more causality to internal factors of the victims than did coworkers, $t(147)=$ $-8.68, p<.001$.

Victims and coworkers. On the external scale, accident victims employed relatively more external attributions than did their workmates, $t(144)=2.43$, 


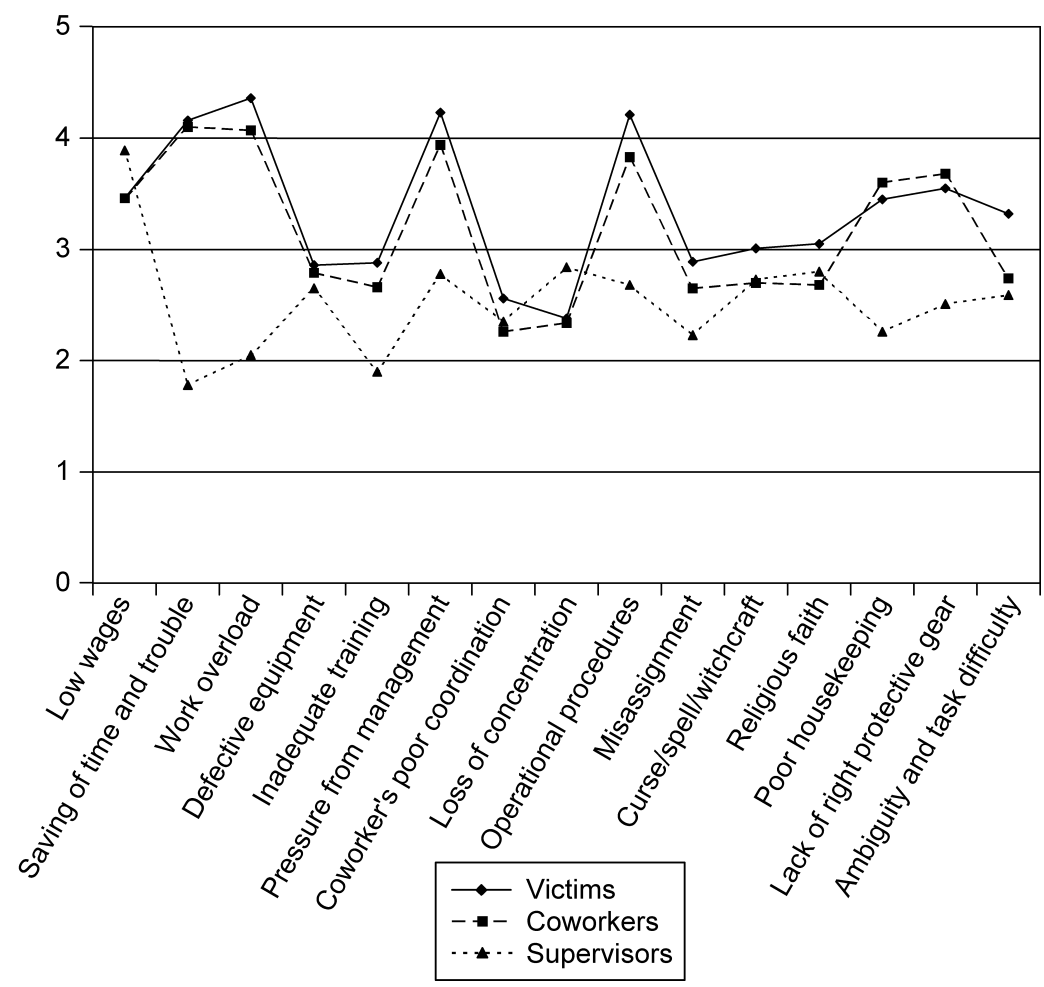

Figure 1. External attributions of Ghanaian accident victims, coworkers, and supervisors.

$p<.001$. On the other hand, they made significantly fewer internal attributions than did their coworkers on the internal scale, $t(217)=-3.19, p<.001$.

Coworkers' attributions on both the internal and external causal factors scales seemed to be midway between those of the victims and their supervisors. They rated victims' responsibility for the accident occurrence lower than did the supervisors, and identified more with the accident victims than with their supervisors. The relationship of all three subgroups in their causality attribution is shown graphically on the two profiles (Figures 1 and 2).

\section{Item-by-Item Comparison}

External causal factors scale. Statistically significant differences between accident victims' and supervisors' attributions were found in 11 of the 15 variables (Table 2). Highly statistically significant differences were recorded between victims' and supervisors' attributions on time saving and trouble saving, 


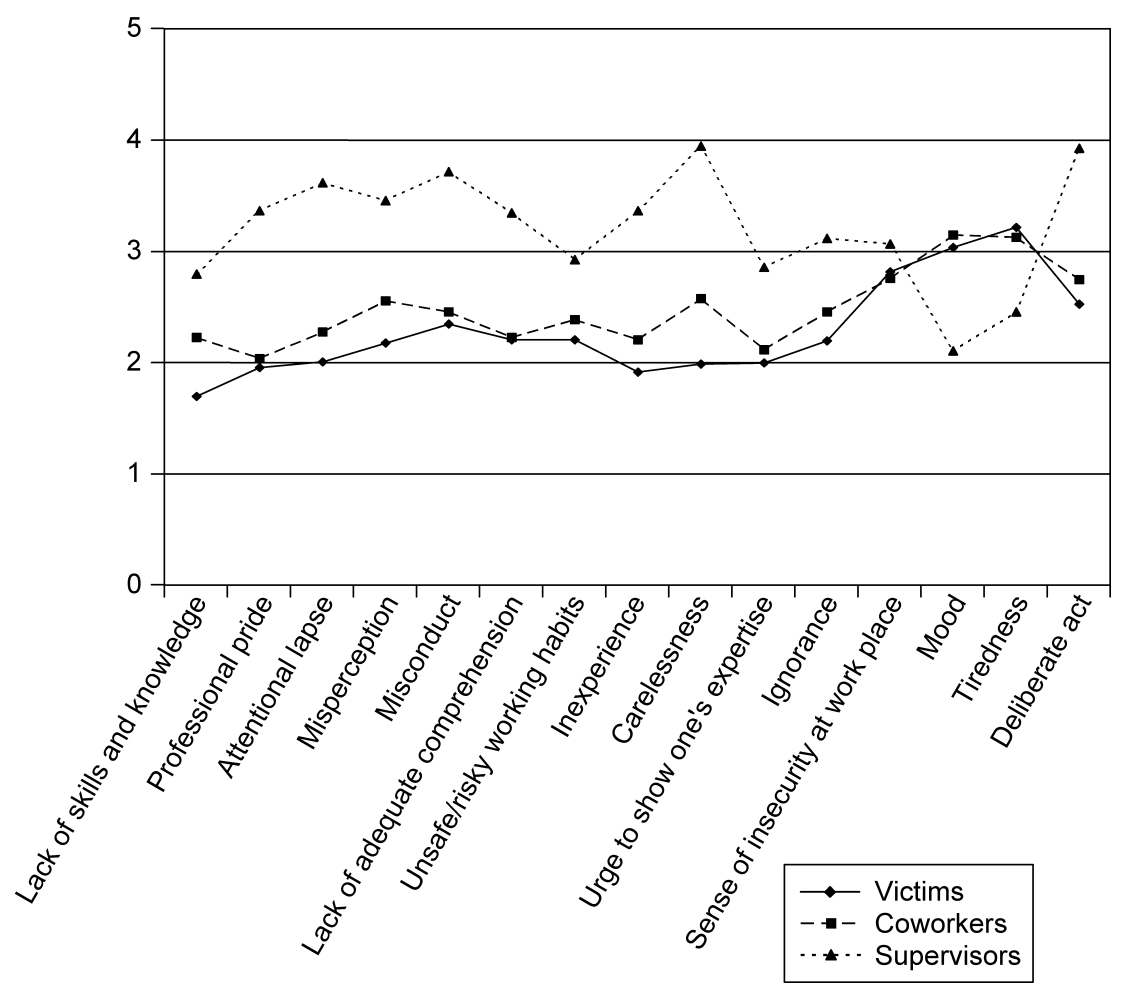

Figure 2. Internal attributions of Ghanaian accident victims, coworkers, and supervisors.

$t(160)=18.97, p<.001$; work overload, $t(125)=18.21, p<.001$; operational procedures (poor working schedules), $t(162)=13.84, p<.001$; and inadequate training, $t(199)=6.38, p<.001$.

Other external causal factors that yielded significant differences were poor housekeeping, $t(190)=7.22, p<.001$; lack of appropriate protective gear, $t(185)=5.53, p<.001$; worker misassignment, $t(194)=3.81, p<.001$; effect of medication, $t(159)=-3.68, p<.001$; and ambiguity and task difficulty, $t(200)=$ $5.36, p<.001$. A statistically significant difference was also found on pressure from management, $t(151)=10.47, p<.001$.

As anticipated, no statistically significant differences were found in attributions to causal factors that did not directly implicate supervisory roles at the workplace. Attributions on religious faith, $t(132)=1.78, n s$; curses, spells, and witchcraft, $t(145)=1.87, n s$; and coworker's poor coordination, $t(189)=1.22, n s$, as causal agents did not show any significant difference, nor was any significance obtained regarding defective equipment, $t(182)=1.11$, $n s$. This latter finding was 
not anticipated. A statistically significant difference was expected as a causal factor that implicated supervisors and management, but diminished the subordinates' responsibility. A relatively smaller, but significant difference was recorded on low wages, $t(201)=-2.45, p<.05$. Regarding attributions to wages and effect of medication, supervisors scored higher than did accident victims.

Internal causal factors scale. Statistically significant differences were found in all but one of the internal causal factors (Table 3). Highly statistically significant differences were recorded between the victims' and supervisors' attributions on lack of skill, $t(125)=-6.55, p<.001$, and professional pride, $t(129)=-8.22$, $p<.001$.

There were also some highly significant differences in workers' attention lapse, $t(172)=-8.74, p<.001$; and misperception, $t(151)=-7.57, p<.001$. Statistically significant differences also were recorded on attributions to misconduct, $t(149)=-7.36, p<.001$; lack of adequate comprehension, $t(143)=-6.68, p<.001$; unsafe and risky working habits, $t(131)=-4.13, p<.001$; and inexperience, which led the worker to misjudge his priorities and to make irrecoverable mistakes, $t(135)=-9.16, p<.001$.

As anticipated, causal factors such as carelessness, $t(146)=-12.63, p<.001$; victims' urge to show off their expertise, $t(143)=-5.26, p<.001$; victims' inability to detect the inherent level of risk, $t(146)=-4.96, p<.001$; and their willful violation and blatant disregard of the company's safety policy, $t(140)=-8.55, p<$ .001 , showed highly significant differences. These are all causal factors that implicated the victims' levels of competence and dedication to work.

Interestingly, causal factors such as mood-had a bad day, $t(189)=5.72, p<$ .001 ; and tiredness and exhaustion, $t(146)=4.19, p<.001$, showed highly significant differences. Understandably, victims scored higher on these internal causal factors than did their supervisors. Not surprisingly, the only internal causal factor that did not show any statistically significant difference between the victims' and the supervisors' attributions concerned the victims' sense of job security, $t(161)=$ $-1.42, n s$. Neither victims nor supervisors considered job insecurity, which tends to impact negatively on employees' job attitudes, work behaviors, and psychological health, and thereby increases their accident susceptibility as important causal factors.

\section{Discussion}

The results show that attributions on both the external and internal causal factors scales were largely self-serving. Both the accident victims and their supervisors tended to attribute the accident occurrence to causal factors that diminished their own roles and responsibilities. What is evident here is supervisors' persistent attribution to workers' dispositional factors, and accident victims' attribution to workplace factors as causal factors for the accident occurrence. 
As stated earlier, self-defensive attribution theory predicted that accident victims would display a consistent pattern of external attributions, and their supervisors would display a consistent pattern of internal attributions. The current results confirm this assumption. It can be argued that this divergence in causality attributions was motivated by self-defensive ambition.

\section{Accident Victims' Perspective}

Accident victims, more than their supervisors, attributed accident causality mostly to workplace and situational factors, and less to dispositional factors. These causal factors exempted them from blame and responsibility, and held supervisors responsible for the accident occurrence. Prominent among these factors were the saving of time and trouble, work overload, inadequate training, pressure from management, operational procedures, poor housekeeping, ambiguity and task difficulty, and lack of suitable protective gear, all of which implied supervisory inefficiency and incompetence.

The fact that attributions were motivated by defensive ambition is supported by their consideration of supernatural and spiritual forces as causality factors. This is evidenced by attributions to witchcraft and religious beliefs. Even though both the accident victims and their supervisors attributed greatly to these factors, comparative analysis of their mean scores on witchcraft (victims, $M=3.03$; supervisors, $M=2.73$ ) and religious beliefs (victims, $M=3.05$; supervisors, $M=$ 2.08) reveals that accident victims, more than their supervisors, employed supernatural and witchcraft attributions as causal factors. According to responsibility attribution theorists, an actor is not deemed responsible for actions that are beyond his volitional control (Helkama, 1981; Weiner, 1995, 1996). Thus, by their witchcraft and sorcery attributions, the accident victims excused themselves from the accident causality and implied that what happened to them was beyond volitional control and could have happened to any worker in that job situation. As Shaffer (1984) has suggested, such witchcraft and sorcery attributions function primarily to satisfy psychological needs to buffer the impact of negative incidents. This line of reasoning, ostensibly, might have been construed as a defense mechanism intended to lead management to the logical conclusion that they, the victims, were not totally responsible for the accident.

Interestingly, the difference was not statistically significant. The reason for this observation could lie in the pervasive fatalistic interpretation of accidents in the Ghanaian culture where accident causation carries a predominantly fatalistic connotation and is usually laden with mystical and supernatural influences. It is a common belief among most Ghanaians that human affairs are controlled by mystical laws or supernatural forces that govern the whole of reality. Such ideas concerning spiritual forces are clearly evident in the explanations given for causes of misfortunes, calamities, illnesses, and death. 
The finding that victims' attributions were motivated by defensive ambitions gets support from the accident literature. The causality attribution of victims in the present study contradicts the overwhelming objective evidence that attributes a high proportion of accidents ( $80 \%$ to $85 \%$ ) to human error (e.g., Heinrich, Petersen, \& Roos, 1980; Salminen \& Tallberg, 1996). Essentially, what these studies have shown is that accident occurrence is a result of both human error and environmental factors, with the role of human factors being considered the main antecedent to the accident process. All other things being equal, the responses of accident victims should at least have reflected the impact of internal factors to a greater extent. Undoubtedly, it can be argued that the environmental factors cited by the accident victims represent causes that are at least partially connected to inadequate supervision or management inefficiency. For example, work environments with good housekeeping have shown a reduction rate of $70 \%$ to $90 \%$ in accident occurrence (e.g., Saari \& Näsänen, 1989). But by their emphatic external attributions and marginal internal attributions, the victims may have biased their attributional explanations in order to effectively counteract any direct blame that would have endangered their work positions.

The reasons inspired the use of these defensive attributions were noted during the field studies. Discussions with top management and safety officials of the various departments that participated in the study revealed that workers were usually penalized to discourage future accident recurrence. This ranges from suspension from work to outright dismissal. Given that no worker would appreciate a deduction from his meager wages, let alone loss of his job, the accident victims might have been tempted to rationalize their undesirable behavior when it entailed the risk of a punitive response from management. Thus, when faced with management's possible negative appraisal for their competence and work ability, the accident victims were motivated to attribute their failings to external factors, and particularly to uncontrollable spiritual forces, so as to lessen their own involvement in the accident occurrence.

As anticipated, the internal causal factors scale showed that, unlike their supervisors, accident victims designated significantly fewer attributions to internal causal factors. Not only were they motivated to invoke external attributions, but they also reported fewer internal attributions. They attributed less to causal factors, such as lack of skill, incompetence, inexperience, ignorance, risky work behavior, misconduct, carelessness, and blatant disregard for the company's safety policies, all of which would have questioned their work competence.

Interestingly, accident victims readily ascribed to themselves such internal causal factors that were perceived to be unavoidable and general to all workers, and therefore posed no danger to their self-esteem and future job prospects. A case in point is the observation on mood, and on tiredness and exhaustion. The victims, more than their supervisors, ascribed more causal importance to these 
internal variables (mood: victims, $M=3.02, S D=1.24$; supervisors, $M=2.08$, $S D=1.07$; tiredness and exhaustion: victims, $M=3.19, S D=1.08$; supervisors, $M=2.43, S D=1.37$ ). Taken together, the recorded causal attributions are consistent with the defensive attribution interpretations in which the accident victims stress external factors and underrate internal attributions that adversely implicate their competence and self-esteem.

\section{Supervisors' Perspective}

The results of the study indicate that supervisors attributed accident causality mostly to factors internal to the victims, and only marginally to workplace factors. The causes were mostly attributed to the workers' lack of skill, carelessness, misconduct, ignorance, inexperience, professional pride, and blatant disregard of the company's safety regulations. These causal factors arose not only from the victims' dispositional characteristics, but more importantly they represented causes that were volitional, controllable, and therefore potentially preventable.

With this line of attribution, victims' work practices tended to be erroneous and deviant, and therefore revealed their stable dispositions. Essentially, the causal factors brought forth by supervisors were the kind that implicated the roles of accident victims, and consequently diminished those of the supervisors in the accident process. Thus, from the supervisors' perspective, the victims' actions were a breach of the underlying social norms concerning workplace safety.

A plausible explanation for this line of attribution could have been that the supervisors might have considered their inferred judgments as the appropriate yardstick in their causal analyses. With the overwhelming evidence that implicates the role of human error in the accident process (e.g., Heinrich et al., 1980; Salminen \& Tallberg, 1996), the supervisors may have been tempted to lay blame on a subordinate worker rather than finding the true underlying reasons. Previous research has established that when observed behavior is consistent with expectations, the perceiver relies on pre-existing causal theories as to its cause (Fiske \& Neuberg, 1990).

Another plausible explanation for the worker-focused attribution recorded by the supervisors could relate to their social status and hierarchical position at the workplace. As part of the managerial staff, supervisors are responsible for conducting accident investigations in their various departments. It therefore seems logical to assume that the supervisors' analyses in the current study were influenced by self-protective and self-defensive biases. Situational and environmental factors that might otherwise have been seen as compelling explanations for the accident occurrence might thus have been discounted and ignored in favor of the workers' dispositional factors. Kouabenan et al. (2001) observed that workers with higher hierarchical positions than those of their subordinate work crew 
tended to place blame and responsibility on the dispositional qualities of the victims. Similarly, DeJoy (1987) noted that supervisors tended to attribute a more causal role to accident victims, even in cases where the causal data defied common logic and organization.

A conceivable explanation for the recorded worker-focused attributions could have been the supervisors' desire to ward off threats of future victimization, and for psychological assurance that an accident of such kind would not happen to them. According to the literature on defensive attributions (Shaver, 1970a; Walster, 1966) the suffering of an innocent victim threatens the observer's belief in his own victimization in a similar accident. The need to believe that the accident is controllable and will not happen to the observer instigates a biased attribution for its occurrence.

In order to protect themselves from feeling vulnerable to such devastating accidents, the supervisors could have been motivated to construct their attributional explanations to correspond to the personal negative characteristics of the accident victims. In essence, the high levels of attributions to internal causal factors in the victims - considered volitional, controllable, and preventable - therefore could have been attempts to deny that such an accident was uncontrollable, unpreventable, and therefore could happen to them also. Thus, by their internal attributions, the supervisors might have felt protected, assuring themselves that they were different persons, with totally different dispositional qualities and would have acted differently in a similar situation.

Additional support for the idea that supervisors' attributions were motivated by self-protective and defensive tendencies comes from analyses of their attributions to factors with less impact on their job competence and self-esteem. These analyses explicitly showed that the supervisors only took recourse to such external causal attributions that had a negative impact on their work performance and threatened their positions, but not to those that implicated top management. For example, the external causal factors scale indicated that causal factors such as wages, workers' poor coordination, and defective equipment showed either a weak or no statistically significant difference between supervisors' and victims' attributions.

Regarding wages, the difference in attributions was small but statistically significant. Interestingly, supervisors scored higher than did their subordinates. Even though supervisors are considered part of management, because they were not responsible for workers' wages, defensiveness was minimal. This also explains the absence of a statistically significant difference on defective equipment and workers' poor coordination. The supervisors distanced themselves from managerial responsibilities of providing monetary allocation for equipment and machinery maintenance. In these cases, neither their self-esteem nor job competence was being threatened. Therefore, self-serving and self-protective attributions were lacking. Apparently, it was only when a situation had affective 
significance for them that the resultant attributions were biased to suit their wishes and desires.

All in all, these results show clearly that the attributions recorded were influenced by self-defensive ambitions. The common thread in the attribution assignment seems to be a tendency toward self-protection from both the accident victims and their supervisors. When evaluating the accident occurrence, both groups applied to it only those inferential attributions that minimized their own roles in the accident process.

\section{Implications of the Findings in the Work Environment}

From a practical perspective, the current findings make important contributions to workplace accident investigation and safety management. According to the literature on industrial accidents and safety, attributions for accident causality are a central part of the formal analysis of workplace hazards and accidents (DeJoy, 1990, 1994). It is from such causal analyses that safety interventions that can forestall future recurrences are developed. The noted divergence in attributions, therefore, can lead to a misdirection of subsequent safety interventions and easily be the origin of organizational conflict. To effectively learn from accidents and provide viable safety interventions, it would be feasible to seek and compare causal explanations of all employees (both subordinates and supervisors) and the beliefs that underlie their causal attributions. Such a perspective of accident causal analysis would create a more responsible and balanced approach to safety, as it will likely address multiple levels simultaneously.

Among the explanations for the relatively numerous defensive attributions proposed by the victims was the poor safety climate in which the management was committed to punitive sanctions for breaches of safety policies. To ensure sound and accurate accident analyses, it will be crucial that accident victims are not routinely subjected to culpability and punishment. A safety climate in which management and supervisors adopt a committed and nonpunitive approach to safety would result in norms of behavior that will encourage workers to be more open about their job-skill deficiencies, and to accept responsibility rather than resort to defensiveness.

The major limitation of this research was participants' need to recall the industrial mishaps. This involved the risk of making responsibility connections in the accident occurrence difficult to infer. Such an error is rather common in the context of industrial accidents that are studied retrospectively. However, our choice of participants who were accident victims, coworkers, and supervisors at their workplace and who were involved in the accident under study less than 1 year before the study hopefully facilitated a fair recall of the accident process. Prospective examinations of accident processes could be viable alternatives to retrospective studies. 
The other limitation relates to the sample composition. The participants were predominantly male. This is because male workers are usually assigned the most dangerous roles in workplaces and therefore are more likely to get involved in serious industrial accidents. This could limit the extent to which the results can be generalized to female workers. Notwithstanding these limitations, the results of this study are consistent with those of most previous studies in this domain (e.g., DeJoy, 1987; Mitchell \& Wood, 1980; Salminen, 1992, 1997).

\section{References}

Blass, T. (1996). The Milgram obedience experiment: Support for a cognitive view of defensive attribution. Journal of Social Psychology, 136, 407-410.

Burger, J. M. (1981). Motivational biases in the attribution of responsibility for an accident: A meta-analysis of the defensive attribution hypothesis. Psychological Bulletin, 90, 496-512.

Chaikin, A., \& Darley, J. M. (1973). Victim or perpetrator? Defensive attribution of responsibility and the need for order and justice. Journal of Personality and Social Psychology, 25, 268-275.

DeJoy, D. M. (1985). Attributional process and hazard control management in industry. Journal of Safety Research, 16, 61-71.

DeJoy, D. M. (1987). Supervisor attributions and responses for multiple workplace accidents. Journal of Occupational Accidents, 9, 213-223.

DeJoy, D. M. (1990). Toward a comprehensive human factors model of workplace accident causation. Professional Safety, 35(5), 11-16.

DeJoy, D. M. (1994). Managing safety in the workplace: An attributional theory analysis and model. Journal of Safety Research, 25, 3-17.

Fiske, S. T., \& Neuberg, S. L. (1990). A continuum of impression formation, from category-based to individual processes: Influences of information and motivation on attention and interpretation. In L. Berkowitz (Ed.), Advances in experimental social psychology (Vol. 23, pp. 1-74). New York, NY: Academic Press.

Gyekye, A. S. (2001). The self-defensive attribution theory revisited: A culturecomparative analysis between Finland and Ghana. Helsinki, Finland: Yliopistopaino.

Heinrich, H. W., Petersen, D., \& Roos, N. (1980). Industrial accident prevention (5th ed.). New York, NY: McGraw-Hill.

Helkama, K. (1981). Toward a cognitive-developmental theory of attribution of responsibility: A critical review of empirical research and some preliminary data. Annales Acedemiae Scitarium Fennicae Dissertationes Humanarum Litterarum 2, 1981.

Hofstede, G. H. (1980). Culture's consequences. Beverly Hills, CA: Sage. 
Hofstede, G. H. (1991). Cultures and organizations: Software of the mind. London, UK: McGraw-Hill.

Kouabenan, D. R. (1985). Degree of involvement in an accident and causal attribution. Journal of Occupational Accidents, 7, 187-194.

Kouabenan, D. R., Gilibert, D., Medina, M., \& Bouzon, F. (2001). Hierarchical position, gender, accident severity, and causal attribution. Journal of Applied Social Psychology, 31, 553-575.

Mitchell, T. R., \& Kalb, L. S. (1981). Effects of outcome knowledge and outcome valence on supervisors' evaluations. Journal of Applied Psychology, 66, 604-612.

Mitchell, T. R., \& Wood, R. E. (1980). Supervisors' responses to subordinate poor performance: A test of an attribution model. Organizational Behavior and Human Performance, 25, 123-138.

Saari, J., \& Näsänen, M. (1989). The effect of positive feedback on industrial housekeeping and accidents: A long-term study at a shipyard. International Journal of Industrial Ergonomics, 4, 201-211.

Salminen, S. (1992). Defensive attribution hypothesis and serious occupational accidents. Psychological Reports, 70, 1195-1199.

Salminen, S. (1997). Risk taking, attributions, and serious accidents. Helsinki, Finland: Finnish Institute of Occupational Health.

Salminen, S., \& Tallberg, T. (1996). Human errors in fatal and serious occupational accidents in Finland. Ergonomics, 39, 980-988.

Shaffer, L. S. (1984). Fatalism as an animistic attribution process. Journal of Mind Behavior, 5, 351-362.

Shaver, K. G. (1970a). Defensive attribution: Effects of severity and relevance on the responsibility assigned for an accident. Journal of Personality and Social Psychology, 14, 101-113.

Shaver, K. G. (1970b). Redress and conscientiousness in the attribution of responsibility for accidents. Journal of Experimental Psychology, 6, 100-110.

Shaw, J. I., \& Skolnick, P. (1971). Attribution of responsibility for a happy accident. Journal of Personality and Social Psychology, 18, 380-383.

Triandis, H. C. (1995). Individualism and collectivism. Boulder, CO: Westview.

Turner, B. A., \& Pidgeon, N. F. (1997). Man-made disasters (2nd ed.). Oxford, UK: Butterworth-Heinmann.

Vidmar, N., \& Crinklaw, L. D. (1974). Attributing responsibility for an accident: A methodological and conceptual critique. Canadian Journal of Behavioural Sciences, 6, 112-130.

Walster, E. (1966). Assignment of responsibility for an accident. Journal of Personality and Social Psychology, 3, 73-79.

Weiner, B. (1995). Judgments of responsibility: A foundation for a theory of social conduct. New York,. NY: Guilford. 
Weiner, B. (1996). Searching for order in social motivation. Psychological Inquiry, 7, 199-216.

Wong, P. T., \& Weiner, B. (1981). When people ask "why" questions, and the heuristics of attributional search. Journal of Personality and Social Psychology, 40, 650-663.

Zuckerman, M. (1979). Attribution of success and failure revisited: The motivational bias is alive and well in attribution theory. Journal of Personality, 47, 245-287. 
Appendix

\section{Questionnaire}

How would you rate the influence of the following factors as contributory factors to the accident?

Response alternatives: $1=$ very little $; 2=$ quite little $; 3=$ neither much nor little (neutral); 4 = quite much; 5 = very much

\section{External Causal Factors}

Low wages

Savings of time and trouble

Work overload (too much to attend to)

Unsafe or defective equipment and facilities

Inadequate training, orientation, and supervision

Pressure from management/supervisor

Coworkers' fault (e.g., poor coordination)

Loss of concentration (effect of medication)

Operational procedures (work and production schedule)

Misassignment (victim was new to the situation/environment)

Being a victim of some curse/spell/witchcraft

Religious beliefs (invincible from accidents)

Poor housekeeping

Lack of right protective equipment and gear

Ambiguity and task difficulty (uncertainty about task demands)

\section{Internal Causal Factors}

Lack of skill and knowledge

Professional pride (underestimated situation)

Lapse in attention

Misperception (poor judgment or assessment of situation)

Misconduct (failure to use protective clothing, improper use of tools, equipment, facilities)

Lack of adequate comprehension and ability to follow prescribed work procedures

Unsafe/risky working habits (prone to accidents)

Inexperience, mistaken priorities, irreconcilable actions

Carelessness (exceeding prescribed limits)

Self-esteem (urge to impress managers and coworkers) 
Ignorance (lack of awareness of hazards involved)

Victim's sense of insecurity at work

Victim just had a bad day

Tiredness/fatigue and reduced alertness

Willful violations (blatant disregard of work procedures and neglect of safe practices) 